\title{
Efeito de Doses Reduzidas de Glyphosate e Paraquat Simulando Deriva na Cultura do Milho
}

\author{
Effect of Reduced Rates of Glyphosate and Paraquat Simulating Drift in Corn Crop
}

MAGALHÃES, P.C. ${ }^{2}$, SILVA, J.B. ${ }^{3}$, DURÃES, F.O.M. ${ }^{2}$, KARAM, D. ${ }^{2}$ e RIBEIRO, L.S ${ }^{4}$

RESUMO - A aplicação de herbicidas, seja para a dissecação de culturas ou para o controle de plantas daninhas, vem crescendo, devido a expansão da fronteira agrícola brasileira. Esse fato aumenta os riscos de ocorrência de deriva acidental em culturas vizinhas suscetíveis. As perdas em produtividade são desconhecidas em muitas situações de ocorrência de deriva de herbicidas. O objetivo deste trabalho foi avaliar a possível toxicidade causada pela deriva de doses reduzidas de dois herbicidas (glyphosate e paraquat) no período inicial de desenvolvimento da cultura do milho. Foram utilizadas cinco doses simulando deriva - 2, 4, 6, 8 e $12 \%$ da dose recomendada ( $1.440 \mathrm{~g} \mathrm{ha}^{-1}$ de glyphosate e $400 \mathrm{~g} \mathrm{ha}^{-1}$ de paraquat) - sobre o cultivar de milho híbrido triplo BRS 3123 . No florescimento, foram avaliados altura da planta, área foliar, peso da matéria seca, teor de clorofila e sintomas visuais de injúria. Na colheita, avaliaram-se estande final, peso de espigas, peso de 1.000 grãos e produção de grãos. A altura das plantas, a área foliar e o peso da matéria seca não foram afetados pelo efeito das derivas nos dois anos agrícolas (1996/97 e 1997/98), exceto pela área foliar, que em 1997/ 98 sofreu redução, sobretudo no tratamento com $12 \%$ da dose normal de glyphosate. De maneira geral, os resultados obtidos para as demais características foram semelhantes nos dois anos de condução do ensaio. O teor de clorofila nas folhas e o estande final não foram afetados pelas doses reduzidas. O grau de toxicidade, avaliado por meio de plantas injuriadas pela deriva, aos 7,14 e 21 dias após a aplicação dos herbicidas, apresentou diferenças significativas. Os maiores danos foram observados com a maior subdose simulando deriva dos herbicidas. O peso de 1.000 grãos não foi afetado, ao passo que a produção de espigas e de grãos foi severamente prejudicada. Observou-se que a deriva simulada dos herbicidas em altas concentrações afetou o desenvolvimento das plantas e reduziu a produção de grãos. Já a aplicação em baixas concentrações ( 2 a 4\%) não afetou o desenvolvimento das plantas nem tampouco a produtividade.

Palavras-chave: toxicidade, herbicidas, dessecantes, Zea mays.

\begin{abstract}
Herbicide application has increased considerably in the Brazilian agriculture over the last years, either for weed management or crop desiccation. The intensive use of herbicide shas brought risks of drift in sensitive neighbor crops. Drift effects on crops susceptible to herbicides is not well quantified in several situations. The objective of this study was to evaluate the injuries caused by simulated herbicide drift on the initial phase of growth of the maize crop. Two herbicides with five concentrations of simulated drift were used: 2, 4, 6, 8 and 12\% of the recommended rate $\left(1,440 \mathrm{~g} \mathrm{ha}^{-1}\right.$ of glyphosate and $400 \mathrm{~g} \mathrm{ha}^{-1}$ of paraquat), sprayed over the top foliage of the maize triple hybrid BRS 3123. Plant height, leaf area, dry weight, leaf chlorophyll content and visual symptoms of injury were evaluated at flowering time. Final stand, ear weight, 1,000 grains weight and grain production. Plant height, leaf area, leaf chlorophyll content and dry weight were not affected by simulated drifts in both years (1996/97 and 1997/98), except the leaf area in 1997/98, which had a reduction especially in the treatment where $12 \%$ of the
\end{abstract}

1 Recebido para publicação em 22/1/2001 e na forma revisada em 2/7/2001.

Trabalho desenvolvido pela Embrapa Milho e Sorgo, Caixa postal 151, Rod. MG 424, Km 65 - 35 701-970 Sete Lagoas-MG.

2 Pesquisadores, Ph.D., Embrapa Milho e Sorgo, Sete Lagoas-MG. ${ }^{3}$ Consultor Autônomo, Ph.D., Sete Lagoas-MG. ${ }^{4}$ Eng ${ }^{\mathrm{a}}$ Agrônoma, M.S., estagiária da Embrapa Milho e Sorgo.

Planta Daninha, Viçosa-MG, v.19, n.2, p.247-253, 2001 
glyphosate rate was used. In general, the results for the other traits were similar in both years in which the trials were carried out. At harvesting time, final stand and weight of 1,000 grains were not affected by simulated drifts, whereas ear weight and grain production were severely affected. The degree of visual injuries evaluated through percentage of plants injured by drift at 7, 14 and 21 days after herbicide application showed significant differences. The major damages were observed with the greater dosage of simulated drift. Weight of 1,000 grains was not affected, whereas ears and grain production were severely affected. Herbicide drift in high concentrations affected the plant development and reduced grain production while application of glyphosate and paraquat in low concentrations (2-4\% of the recommended rate) did not affect plant development nor grain production.

Key words: toxicity, herbicides, desiccation, Zea mays.

\section{INTRODUÇÃO}

A utilização de herbicidas dessecantes nos dias atuais é cada vez maior, principalmente com o advento do plantio direto (Puríssimo, 1999). Dentre os herbicidas mais utilizados para essa prática destacam-se o glyphosate e o paraquat. O glyphosate é considerado um inibidor da biossintese de aminoácidos essenciais de cadeia aromática, enquanto o paraquat é um inibidor do fotossistema I ou formador de radicais livres durante a fotossintese (Puríssimo, 1999). Além do mais, o controle de plantas daninhas por meio do uso de herbicidas não-seletivos tornou-se uma prática tão difundida nas lavouras, que é quase uma rotina obrigatória para os agricultores (Silva et al., 1998). É comum observar neste tipo de manejo cultural a ocorrência de derivas, as quais podem afetar uma lavoura vizinha, sobretudo quando a aplicação é realizada sob condição de vento ou outras condições de ambiente que favoreçam a sua volatilização e posterior deposição foliar dos herbicidas utilizados (Wall, 1994). Esse fato pode prejudicar o desenvolvimento das plantas e, conseqüentemente, a produtividade de grãos, uma vez que a área foliar verde é a principal fonte de fotoassimilados para a cultura do milho (Magalhães et al., 1995; Magalhães \& Jones, 1990b).

O plantio do milho está atualmente difundido em todo o país (Milho 1998), aumentando assim as possibilidades de ser atingido por uma deriva oriunda de lavoura vizinha. Na atualidade, o efeito das derivas assume importância ainda maior, devido à crescente preocupação e conscientização da população quanto a questões ambientais. Diversos estudos têm sido conduzidos para melhorar a eficiência da aplicação, porém até hoje não se dispõe de nenhuma técnica capaz de garantir ou manter completamente os agrotóxicos dentro das áreas-alvo (Bode, 1984). Portanto, o estudo de derivas simuladas, com seus conseqüentes efeitos na cultura do milho, torna-se de extrema importância. Ademais, poucas são as informações disponiveis na literatura capazes de quantificar prejuízos decorrentes da deriva de herbicidas tóxicos ao milho. Os questionamentos com relação a possiveis prejuízos são muitos. Por exemplo: A "queima" da parte aérea pode afetar a produtividade? A planta se recupera? Quais são, enfim, as conseqüências da deriva?

Dentro desse contexto, o objetivo deste trabalho foi avaliar as injúrias causadas por doses reduzidas dos herbicidas glyphosate e paraquat simulando deriva e os seus efeitos na produção final de grãos.

\section{MATERIAL E MÉTODOS}

O ensaio foi conduzido na área experimental do Centro Nacional de Pesquisa de Milho e Sorgo (CNPMS), Sete Lagoas - MG, durante dois anos agrícolas (1996/97 e 1997/98). O solo utilizado foi um Latossolo Vermelho-Escuro, fase cerrado, textura argilosa, adubado de acordo com a recomendação da análise do solo. Foram aplicados, em ambos os anos agrícolas, $300 \mathrm{~kg} \mathrm{ha}^{-1} \mathrm{da}$ fórmula 8-28-16 + zinco e $90 \mathrm{~kg} \mathrm{ha}^{-1}$ de $\mathrm{N}$ em cobertura. A área experimental foi irrigada sempre que necessário, baseando-se em leituras de tensiômetros instalados previamente a 20 e $40 \mathrm{~cm}$ de 
profundidade. Adotou-se o limite de -0,07 MPa como o momento da irrigação (Resende et al., 1992). Foi utilizado o milho híbrido triplo BRS 3123, nos seguintes tratamentos:

1 - Testemunha absoluta

2 - $2 \%$ da dose recomendada de glyphosate $\left(28,8 \mathrm{~g} \mathrm{ha}^{-1}\right)$

3 - 4\% da dose recomendada de glyphosate $\left(57,6 \mathrm{~g} \mathrm{ha}^{-1}\right)$

4 - $6 \%$ da dose recomendada de glyphosate $\left(86,4 \mathrm{~g} \mathrm{ha}^{-1}\right)$

5 - 8\% da dose recomendada de glyphosate (115,2 $\left.\mathrm{g} \mathrm{ha}^{-1}\right)$

6 - 12\% da dose recomendada de glyphosate $\left(172,8 \mathrm{~g} \mathrm{ha}^{-1}\right)$

7 - Testemunha com adjuvante (Agral)

8 - $2 \%$ da dose recomendada de paraquat ( $\left.8 \mathrm{~g} \mathrm{ha}^{-1}\right)$

9 - 4\% da dose recomendada de paraquat ( $\left.16 \mathrm{~g} \mathrm{ha}^{-1}\right)$

10 - $6 \%$ da dose recomendada de paraquat ( $\left.24 \mathrm{~g} \mathrm{ha}^{-1}\right)$

11 - 8\% da dose recomendada de paraquat (32 $\mathrm{g} \mathrm{ha}^{-1}$ )

12 - 12\% da dose recomendada de paraquat ( $48 \mathrm{~g} \mathrm{ha}^{-1}$ )

Os produtos comerciais utilizados no ensaio foram: Roundup (glyphosate), gramoxone (paraquat) em mistura no tanque com Agral a $0,03 \% \mathrm{v} / \mathrm{v}$.

As derivas foram simuladas em várias concentrações, tomando-se como base a dose recomendada do respectivo herbicida. Assim, para o glyphosate, cuja dose recomendada é de até $41 \mathrm{ha}^{-1}$, as derivas foram de: $80 \mathrm{ml} \mathrm{ha}^{-1}$, que corresponde a $2 \%$ da dose utilizada; $160 \mathrm{ml} \mathrm{ha}{ }^{-1}$ (4\%); $240 \mathrm{ml} \mathrm{ha-1}(6 \%) ; 320 \mathrm{ml} \mathrm{ha}^{-1}$ (8\%); e $480 \mathrm{ml} \mathrm{ha}^{-1}$ (12\%). O paraquat mais adjuvante (Agral a 0,03\% v/v), cuja dose recomendada é de $2 \mathrm{~L} \mathrm{ha}^{-1}$, foi utilizado nas seguintes concentrações: $40 \mathrm{ml} \mathrm{ha}^{-1}$, que corresponde a $2 \%$ da dose recomendada; $80 \mathrm{ml} \mathrm{ha}^{-1}(4 \%)$, $120 \mathrm{ml} \mathrm{ha}^{-1}$ (6\%); $160 \mathrm{ml} \mathrm{ha}^{-1}$ (8\%); e $240 \mathrm{ml} \mathrm{ha}^{-1}$ (12\%). A esses tratamentos foram adicionadas duas testemunhas: uma absoluta e outra com apenas adjuvante. Essas diferentes concentrações foram pulverizadas sobre as plantas quando o milho apresentava seis folhas completamente desenvolvidas.

A pulverização foi feita em área total, utilizando-se um pulverizador equipado com barra de oito bicos APJ-110.R, pressão de $2,75 \mathrm{kgf} \mathrm{cm}^{-2}$, com o volume de aplicação de $240 \mathrm{~L} \mathrm{ha}^{-1}$. A pressão de pulverização foi obtida através de um cilindro de $\mathrm{CO}_{2}$ e controlada por um manômetro de baixa pressão. As condições climáticas para o ano agrícola 1996/97, no período da aplicação, foram: temperatura média em torno de $22^{\circ} \mathrm{C}$ e umidade relativa de $71 \%$. No ano seguinte, no mesmo período, foram registrados $23^{\circ} \mathrm{C}$ de temperatura e umidade relativa de $81 \%$. Para evitar o efeito da interferência da planta daninha sobre a cultura, mascarando o efeito de injúrias químicas sobre a produtividade, todas as parcelas foram capinadas manualmente, sempre que necessário. Neste trabalho não foi avaliado o efeito dos tratamentos químicos sobre as plantas daninhas.

O delineamento experimental utilizado foi o de blocos ao acaso, com 12 tratamentos e quatro repetições. Os parâmetros avaliados no florescimento foram: altura da planta, área foliar, peso da matéria seca e teor de clorofila. Aos 7, 14 e 21 dias após a aplicação da deriva, foi avaliado o grau de toxicidade das plantas, que corresponde à porcentagem de plantas injuriadas pelos tratamentos. Finalmente, na colheita foram avaliados: estande final, índice de espiga, peso de espigas e grãos e peso de 1.000 grãos.

A parcela experimental foi constituída de quatro linhas de $9 \mathrm{~m}$ de comprimento, espaçadas de $0,90 \mathrm{~m}$, com cinco sementes por metro. Como área útil foram consideradas as duas fileiras centrais de $8 \mathrm{~m}$ de comprimento, abandonando-se 0,50 $\mathrm{m}$ nas extremidades das parcelas. Os dados foram submetidos à análise de variância, e as médias, comparadas pelo teste de Tukey. Os pesos de espigas e de grãos foram corrigidos para a umidade-padrão de $13 \%$.

\section{RESULTADOS E DISCUSSÃO}

No ano agrícola 1996/97, as variáveis relacionadas ao crescimento e desenvolvimento das plantas (altura, área foliar e matéria seca) não foram afetadas pelas doses reduzidas dos herbicidas (Tabela 1). Este resultado discorda do trabalho de Ali \& Fletcher (1978), os quais mostraram que subdoses maiores de glyphosate $\left(280,560,1.120\right.$ e $\left.2.240 \mathrm{~g} \mathrm{ha}^{-1}\right)$ inibiram o crescimento de plântulas de milho e reduziram drasticamente a altura das plantas, o peso fresco e o tamanho das folhas. O teor de clorofila e o estande final, igualmente, não apresentaram diferenças estatisticamente significativas entre os diversos tratamentos (Tabela 2). O grau de toxicidade, que avalia a porcentagem de plantas injuriadas pela deriva, apresentou diferenças estatisticamente significativas. Assim, pode-se observar que o maior grau de danos foi provocado pelas derivas 
simuladas de $12 \%$ da dose recomendada de paraquat e $12 \%$ de glyphosate (tratamentos de número 12 e 6, respectivamente). Estes tratamentos provocaram injúrias em cerca de $49 \%$ das plantas, no caso do paraquat, e $40 \%$ para o glyphosate. Os demais tratamentos resultaram em porcentagem de injúria que decresceu à medida que a porcentagem de dose também decrescia, e o efeito do paraquat parece ter sido mais evidente que o do glyphosate (Tabela 2). $\mathrm{O}$ fato de se verificarem danos mesmo em pequenas doses reduzidas (baixa concentração da deriva) deve-se, provavelmente, ao modo de ação do paraquat, o qual está relacionado à catalisação da reação de síntese de $\mathrm{H}_{2} \mathrm{O}_{2}$ (peróxido de hidrogênio). Quantidades extremamente pequenas de $\mathrm{H}_{2} \mathrm{O}_{2}$ dentro do cloroplasto das plantas são suficientes para destruí-lo e causar danos (Puríssimo, 1999).

Os efeitos observados na parte aérea influíram negativamente no rendimento final de grãos (Tabela 3). Dessa forma, pode-se observar que tanto o peso de espigas como o de grãos foram afetados pela deriva dos herbicidas paraquat e glyphosate na concentração de $12 \%$ da dose recomendada. O peso de espigas e grãos foi reduzido, em relação à testemunha absoluta, cerca de $11 \%$ com a aplicação do glyphosate e $18 \%$ com deriva do herbicida paraquat. $\mathrm{O}$ tratamento que resultou em maior produção de grãos foi a aplicação de $4 \%$ da dose de glyphosate. $\mathrm{O}$ fato de este tratamento ter se destacado dentre os demais não condiz com o seu modo de ação, o qual é responsável pelo esgotamento dos aminoácidos de cadeia aromática, como o triptofano, a fenilalanina e a tirosina, essenciais à síntese de proteína e outras rotas biossintéticas que levam ao crescimento (Puríssimo, 1999). Portanto, o que se pode depreender, inclusive examinando o grau de fitotoxidade, que foi pequeno para esse caso (Tabela 2), é que esta subdose de glyphosate não chegou a afetar as plantas de milho. Resultado semelhante foi reportado por Hoagland et al. (1979), os quais não observaram alterações no teor de proteínas em milho após tratamento com doses reduzidas de glyphosate nas concentrações de $10^{-4}, 5 \times 10^{-4}$ e $10^{-3} \mathrm{M}$. Apesar de a deriva simulada dos herbicidas ter causado alguma toxicidade nas plantas de milho da maioria dos tratamentos, sobretudo aqueles com concentrações de deriva acima de $8 \%$ da dose recomendada, o milho foi capaz de compensar essa perda e, ainda, alcançar bom rendimento de grãos (Tabela 3). Isto se deve provavelmente à redistribuição de carboidratos acumulados no colmo da planta, conforme se verifica no trabalho de Magalhães et al. (1998).

Tabela 1 - Altura de plantas, área foliar e peso da matéria seca de plantas de milho submetidas aos diferentes tratamentos estudados, no ensaio 1996/97. Embrapa Milho e Sorgo. Sete Lagoas-MG

\begin{tabular}{|c|c|c|c|}
\hline Tratamentos ${ }^{\underline{2}}$ & $\begin{array}{l}\text { Altura } \\
(\mathrm{m})\end{array}$ & $\begin{array}{c}\text { Área foliar } \\
\left(\mathrm{cm}^{2}\right)\end{array}$ & $\begin{array}{c}\text { Matéria seca } \\
(\mathrm{g})\end{array}$ \\
\hline 1 - Testemunha & $2,00 \mathrm{a}^{\mathrm{1}^{\prime}}$ & $5.268 \mathrm{a}$ & $140 \mathrm{a}$ \\
\hline 2 - Glyphosate $28,8 \mathrm{~g} \mathrm{ha}^{-1}$ & $1,99 \mathrm{a}$ & $5.341 \mathrm{a}$ & $155 \mathrm{a}$ \\
\hline 3 - Glyphosate $57,6 \mathrm{~g} \mathrm{ha}^{-1}$ & $1,98 \mathrm{a}$ & $5.368 \mathrm{a}$ & $150 \mathrm{a}$ \\
\hline 4 - Glyphosate $86,4 \mathrm{~g} \mathrm{ha}^{-1}$ & $1,96 \mathrm{a}$ & $5.103 \mathrm{a}$ & $142 \mathrm{a}$ \\
\hline 5 - Glyphosate $115,2 \mathrm{~g} \mathrm{ha}^{-1}$ & $1,99 \mathrm{a}$ & $4.361 \mathrm{a}$ & $117 \mathrm{a}$ \\
\hline 6 - Glyphosate $172,8 \mathrm{~g} \mathrm{ha}^{-1}$ & $1,88 \mathrm{a}$ & $4.393 \mathrm{a}$ & $129 \mathrm{a}$ \\
\hline 7 - Testemunha com adjuvante & $2,02 \mathrm{a}$ & $5.086 \mathrm{a}$ & $135 \mathrm{a}$ \\
\hline 8 - Paraquat $8 \mathrm{~g} \mathrm{ha}^{-1}$ & $2,03 \mathrm{a}$ & $5.713 \mathrm{a}$ & $155 \mathrm{a}$ \\
\hline 9 - Paraquat $16 \mathrm{~g} \mathrm{ha}^{-1}$ & $2,10 \mathrm{a}$ & $5.254 \mathrm{a}$ & $132 \mathrm{a}$ \\
\hline 10 - Paraquat $24 \mathrm{~g} \mathrm{ha}^{-1}$ & $1,96 \mathrm{a}$ & $5.161 \mathrm{a}$ & $123 \mathrm{a}$ \\
\hline 11 - Paraquat $32 \mathrm{~g} \mathrm{ha}^{-1}$ & $1,92 \mathrm{a}$ & $4.679 \mathrm{a}$ & $109 \mathrm{a}$ \\
\hline 12 - Paraquat $48 \mathrm{~g} \mathrm{ha}^{-1}$ & $1,94 \mathrm{a}$ & $5.118 \mathrm{a}$ & $125 \mathrm{a}$ \\
\hline $\mathrm{CV}(\%)$ & 6,98 & 12,03 & 13,74 \\
\hline Valor de F & 0,66 & 1,73 & 2,61 \\
\hline DMS & 0,35 & 1.524 & 46 \\
\hline
\end{tabular}

${ }^{1 /}$ Médias seguidas pela mesma letra não diferem entre si, a 5\% de probabilidade, pelo teste de Tukey.

Tabela 2 - Teor de clorofila nas folhas, grau de toxicidade (média de três leituras) e estande final das plantas de milho submetidas aos diferentes tratamentos estudados, no ensaio 1996/97. Embrapa Milho e Sorgo. Sete Lagoas-MG

\begin{tabular}{|l|c|c|c|}
\hline \multicolumn{1}{|c|}{ Tratamentos } & $\begin{array}{c}\text { Clorofila } \\
\left(\mu \mathrm{g} \mathrm{g}^{-1}\right)\end{array}$ & $\begin{array}{c}\text { Grau de } \\
\text { toxicidade } \\
(\%)\end{array}$ & $\begin{array}{c}\text { Estande } \\
\text { final } \\
\left(\mathrm{n}^{\mathrm{o}}\right)\end{array}$ \\
\hline 1 - Testemunha & $2.311 \mathrm{a}^{\mathrm{i}}$ & $0,00 \mathrm{f}$ & $78 \mathrm{a}$ \\
\hline 2 - Glyphosate $28,8 \mathrm{~g} \mathrm{ha}^{-1}$ & $2.253 \mathrm{a}$ & $3,14 \mathrm{ef}$ & $76 \mathrm{a}$ \\
\hdashline 3 - Glyphosate $57,6 \mathrm{~g} \mathrm{ha}^{-1}$ & $2.463 \mathrm{a}$ & $3,30 \mathrm{ef}$ & $77 \mathrm{a}$ \\
\hline 4 - Glyphosate $86,4 \mathrm{~g} \mathrm{ha}^{-1}$ & $2.420 \mathrm{a}$ & $7,98 \mathrm{ef}$ & $76 \mathrm{a}$ \\
\hline 5 - Glyphosate $115,2 \mathrm{~g} \mathrm{ha}^{-1}$ & $2.394 \mathrm{a}$ & $21,88 \mathrm{~cd}$ & $76 \mathrm{a}$ \\
\hline 6 - Glyphosate $172,8 \mathrm{~g} \mathrm{ha}^{-1}$ & $2.475 \mathrm{a}$ & $39,55 \mathrm{ab}$ & $77 \mathrm{a}$ \\
\hline 7 - Testemunha com adjuvante & $2.374 \mathrm{a}$ & $2,38 \mathrm{ef}$ & $75 \mathrm{a}$ \\
\hline 8 - Paraquat $8 \mathrm{~g} \mathrm{ha}^{-1}$ & $2.589 \mathrm{a}$ & $11,90 \mathrm{de}$ & $76 \mathrm{a}$ \\
\hline 9 - Paraquat $16 \mathrm{~g} \mathrm{ha}^{-1}$ & $2.475 \mathrm{a}$ & $19,38 \mathrm{~cd}$ & $78 \mathrm{a}$ \\
\hline 10 - Paraquat $24 \mathrm{~g} \mathrm{ha}^{-1}$ & $2.442 \mathrm{a}$ & $29,55 \mathrm{bc}$ & $78 \mathrm{a}$ \\
\hline 11 - Paraquat $32 \mathrm{~g} \mathrm{ha}^{-1}$ & $2.583 \mathrm{a}$ & $38,30 \mathrm{~b}$ & $75 \mathrm{a}$ \\
\hline 12 - Paraquat $48 \mathrm{~g} \mathrm{ha}^{-1}$ & $2.352 \mathrm{a}$ & $48,72 \mathrm{a}$ & $76 \mathrm{a}$ \\
\hline CV \% & 14,86 & 22,04 & 2,80 \\
\hline Valor de F & 0,31 & 65,52 & 1,22 \\
\hline DMS & 902 & 10,38 & 5,36 \\
\hline
\end{tabular}

1/ Médias seguidas pela mesma letra não diferem entre si, a 5\% de probabilidade, pelo teste de Tukey. 
Tabela 3 - Peso de espiga, produtividade e peso de 1.000 grãos para plantas de milho submetidas aos diferentes tratamentos estudados, no ensaio 1996/97. Embrapa Milho e Sorgo. Sete Lagoas-MG

\begin{tabular}{|c|c|c|c|}
\hline Tratamentos & $\begin{array}{c}\text { Peso de } \\
\text { espigas } \\
\left(\mathrm{kg} \mathrm{ha}^{-1}\right)\end{array}$ & $\begin{array}{c}\text { Produti- } \\
\text { vidade } \\
\left(\mathrm{kg} \mathrm{ha}^{-1}\right)\end{array}$ & $\begin{array}{c}\text { Peso de } \\
1.000 \text { grãos } \\
\text { (g) }\end{array}$ \\
\hline 1 - Testemunha & $5.045 \mathrm{ab}^{1 /}$ & $4.048 \mathrm{ab}$ & $180 \mathrm{a}$ \\
\hline 2- Glyphosate $28,8 \mathrm{~g} \mathrm{ha}^{-1}$ & $5.131 \mathrm{ab}$ & $4.109 \mathrm{ab}$ & $186 \mathrm{a}$ \\
\hline 3 - Glyphosate $57,6 \mathrm{~g} \mathrm{ha}^{-1}$ & $5.669 \mathrm{a}$ & $4.557 \mathrm{a}$ & $189 \mathrm{a}$ \\
\hline 4 - Glyphosate $86,4 \mathrm{~g} \mathrm{ha}^{-1}$ & $5.065 \mathrm{ab}$ & $4.071 \mathrm{ab}$ & $180 \mathrm{a}$ \\
\hline 5 - Glyphosate $115,2 \mathrm{~g} \mathrm{~h}^{-1}$ & $5.171 \mathrm{ab}$ & $4.129 \mathrm{ab}$ & $188 \mathrm{a}$ \\
\hline 6 - Glyphosate $172,8 \mathrm{~g} \mathrm{ha}^{-1}$ & $4.183 \mathrm{c}$ & $3.309 \mathrm{c}$ & $185 \mathrm{a}$ \\
\hline 7 - Testemunha com adjuvante & $5.142 \mathrm{ab}$ & $4.066 \mathrm{ab}$ & $182 \mathrm{a}$ \\
\hline 8 - Paraquat $8 \mathrm{~g} \mathrm{ha}^{-1}$ & $5.133 \mathrm{ab}$ & $4.159 \mathrm{ab}$ & $180 \mathrm{a}$ \\
\hline 9 - Paraquat $16 \mathrm{~g} \mathrm{ha}^{-1}$ & $5.331 \mathrm{ab}$ & $4.288 \mathrm{ab}$ & $190 \mathrm{a}$ \\
\hline 10 - Paraquat $24 \mathrm{~g} \mathrm{ha}^{-1}$ & $4.853 \mathrm{abc}$ & $3.918 \mathrm{abc}$ & $181 \mathrm{a}$ \\
\hline 11 - Paraquat $32 \mathrm{~g} \mathrm{ha}^{-1}$ & $5.003 \mathrm{abc}$ & $4.018 \mathrm{ab}$ & $186 \mathrm{a}$ \\
\hline 12 - Paraquat $48 \mathrm{~g} \mathrm{ha}^{-1}$ & $4.533 \mathrm{bc}$ & $3.595 \mathrm{bc}$ & $191 \mathrm{a}$ \\
\hline $\mathrm{CV} \%$ & 6,73 & 6,93 & 6,02 \\
\hline Valor de $\mathrm{F}$ & 4,93 & 5,15 & 0,52 \\
\hline DMS & 845 & 697 & 27,82 \\
\hline
\end{tabular}

1/ Médias seguidas pela mesma letra não diferem entre si, a 5\% de probabilidade, pelo teste de Tukey.

A área foliar é uma característica importante para se determinar o tamanho da fonte supridora de fotoassimilados (Magalhães et al., 1995). A redução na área foliar pode acarretar problemas para a planta durante o período de enchimento de grãos, uma vez que as folhas, juntamente com o colmo, são a principal fonte para satisfazer, neste período, a alta demanda dos grãos (Magalhães \& Jones, 1990b; Magalhães et al., 1998). Magalhães et al. (1999), confirmando os dados experimentais de Allison \& Watson (1966) e MacKinnon (1979), concluíram que, em condições desfavoráveis, como as de alguns tratamentos do trabalho, o colmo tem maior participação do que em ambientes favoráveis, ou seja, maior quantidade de fotoassimilados é alocada do colmo para o grão em situação de estresse, em que as folhas são menos saudáveis.

No ano agrícola 1997/98, a altura das plantas e o peso da matéria seca não foram afetados pelo efeito da deriva dos dois herbicidas (Tabela 4). A área foliar sofreu redução apenas no tratamento com $12 \%$ da dose recomendada de glyphosate. Os demais tratamentos provocaram ligeira redução na área foliar, em relação à testemunha, porém sem significância estatística (Tabela 4). O teor de clorofila nas folhas e o estande final de plantas não foram afetados pelas doses reduzidas dos dois herbicidas. O grau de fitotoxicidade apresentou diferenças altamente significativas (Tabela 5). Observa-se que os maiores danos visuais nas plantas ocorreram nos tratamentos com 12\% de glyphosate e paraquat. A partir daí, os danos foram sendo gradualmente reduzidos à medida que se diminuía a concentração das derivas. Com relação aos parâmetros de produtividade, apenas o peso de 1.000 grãos não foi afetado (Tabela 6). A produção de espigas e de grãos foi severamente prejudicada, provavelmente devido à perda de área foliar verde provocada pela ação tóxica do herbicida (Magalhães \& Jones, 1990a). Os percentuais de redução na produção de espigas e grãos, com a aplicação das maiores subdoses de glyphosate e paraquat, foram de 29 e $15 \%$, respectivamente (Tabela 6).

Tabela 4 - Altura de plantas, área foliar e peso da matéria seca de plantas de milho submetidas aos diferentes tratamentos estudados, no ensaio 1997/98. Embrapa Milho e Sorgo. Sete Lagoas-MG

\begin{tabular}{|c|c|c|c|}
\hline Tratamentos & $\begin{array}{l}\text { Altura } \\
(\mathrm{m})\end{array}$ & $\begin{array}{l}\text { Área foliar } \\
\left(\mathrm{cm}^{2}\right)\end{array}$ & $\begin{array}{c}\text { Matéria seca } \\
(\mathrm{g})\end{array}$ \\
\hline 1 - Testemunha & $2,30 \mathrm{a}^{\mathbf{1}^{/}}$ & $6.456 \mathrm{a}$ & $233 \mathrm{a}$ \\
\hline 2 - Glyphosate $28,8 \mathrm{~g} \mathrm{ha}^{-1}$ & $2,29 \mathrm{a}$ & $6.188 \mathrm{ab}$ & $226 \mathrm{a}$ \\
\hline 3 - Glyphosate $57,6 \mathrm{~g} \mathrm{ha}^{-1}$ & $2,16 \mathrm{a}$ & $6.825 \mathrm{a}$ & $231 \mathrm{a}$ \\
\hline 4 - Glyphosate $86,4 \mathrm{~g} \mathrm{ha}^{-1}$ & $2,24 \mathrm{a}$ & $5.709 \mathrm{ab}$ & $209 \mathrm{a}$ \\
\hline 5 - Glyphosate $115,2 \mathrm{~g} \mathrm{ha}^{-1}$ & $2,24 \mathrm{a}$ & $5.262 \mathrm{ab}$ & 209 a \\
\hline 6 - Glyphosate $172,8 \mathrm{~g} \mathrm{ha}^{-1}$ & $2,08 \mathrm{a}$ & $3.310 \mathrm{~b}$ & $159 \mathrm{a}$ \\
\hline 7 - Testemunha com adjuvante & $2,30 \mathrm{a}$ & $6.870 \mathrm{a}$ & $248 \mathrm{a}$ \\
\hline 8 - Paraquat $8 \mathrm{~g} \mathrm{ha}^{-1}$ & $2,27 \mathrm{a}$ & $6.316 \mathrm{a}$ & $212 \mathrm{a}$ \\
\hline 9 - Paraquat $16 \mathrm{~g} \mathrm{ha}^{-1}$ & $2,19 \mathrm{a}$ & $4.558 \mathrm{ab}$ & $192 \mathrm{a}$ \\
\hline 10 - Paraquat $24 \mathrm{~g} \mathrm{ha}^{-1}$ & $2,22 \mathrm{a}$ & $5.267 \mathrm{ab}$ & $184 \mathrm{a}$ \\
\hline 11 - Paraquat $32 \mathrm{~g} \mathrm{ha}^{-1}$ & $2,23 \mathrm{a}$ & $5.838 \mathrm{ab}$ & $203 \mathrm{a}$ \\
\hline 12 - Paraquat $\mathrm{g} \mathrm{ha}^{-1}$ & $2,12 \mathrm{a}$ & $5.592 \mathrm{ab}$ & $202 \mathrm{a}$ \\
\hline $\mathrm{CV}(\%)$ & 5,78 & 20,26 & 20,22 \\
\hline Valor de F & 1,23 & 3,08 & 1,29 \\
\hline DMS & 0,32 & 2.879 & 106 \\
\hline
\end{tabular}

1/ Médias seguidas pela mesma letra não diferem entre si, a $5 \%$ de probabilidade, pelo teste de Tukey.

Os resultados verificados para o ano agrícola 1997/98 foram semelhantes aos do ano 1996/97. 
Tabela 5 - Teor de clorofila nas folhas, grau de toxicidade (média de três leituras) e estande final de plantas de milho submetidas aos diferentes tratamentos estudados, no ensaio 1997/98. Embrapa Milho e Sorgo. Sete Lagoas-MG

\begin{tabular}{|l|c|c|c|}
\hline \multicolumn{1}{|c|}{ Tratamentos } & $\begin{array}{c}\text { Clorofila } \\
\left(\mu \mathrm{g} \mathrm{g}^{-1}\right)\end{array}$ & $\begin{array}{c}\text { Grau de } \\
\text { toxicidade } \\
(\%)\end{array}$ & $\begin{array}{c}\text { Estande } \\
\text { final } \\
\left(\mathrm{n}^{\circ}\right)\end{array}$ \\
\hline 1 - Testemunha & $2.346 \mathrm{a}^{\mathrm{1}}$ & $0,48 \mathrm{f}$ & $91 \mathrm{a}$ \\
\hline 2 - Glyphosate $28,8 \mathrm{~g} \mathrm{ha}^{-1}$ & $2.841 \mathrm{a}$ & $9,91 \mathrm{ef}$ & $86 \mathrm{a}$ \\
\hline 3 - Glyphosate $57,6 \mathrm{~g} \mathrm{ha}^{-1}$ & $2.725 \mathrm{a}$ & $1,41 \mathrm{f}$ & $89 \mathrm{a}$ \\
\hline 4 - Glyphosate $86,4 \mathrm{~g} \mathrm{ha}^{-1}$ & $2.517 \mathrm{a}$ & $9,83 \mathrm{ef}$ & $91 \mathrm{a}$ \\
\hline 5 - Glyphosate $115,2 \mathrm{~g} \mathrm{ha}^{-1}$ & $2.527 \mathrm{a}$ & $21,63 \mathrm{cde}$ & $85 \mathrm{a}$ \\
\hline 6 - Glyphosate $172,8 \mathrm{~g} \mathrm{ha}^{-1}$ & $2.518 \mathrm{a}$ & $58,03 \mathrm{a}$ & $82 \mathrm{a}$ \\
\hline 7 - Testemunhana com adjuvante & $2.540 \mathrm{a}$ & $1,28 \mathrm{f}$ & $89 \mathrm{a}$ \\
\hline 8 - Paraquat $8 \mathrm{~g} \mathrm{ha}^{-1}$ & $2.552 \mathrm{a}$ & $17,83 \mathrm{def}$ & $88 \mathrm{a}$ \\
\hline 9 - Paraquat $16 \mathrm{~g} \mathrm{ha}^{-1}$ & $2.783 \mathrm{a}$ & $26,63 \mathrm{cde}$ & $85 \mathrm{a}$ \\
\hline 10 - Paraquat $24 \mathrm{~g} \mathrm{ha}^{-1}$ & $2.562 \mathrm{a}$ & $29,98 \mathrm{~cd}$ & $87 \mathrm{a}$ \\
\hline 11 - Paraquat $32 \mathrm{~g} \mathrm{ha}^{-1}$ & $2.682 \mathrm{a}$ & $38,61 \mathrm{bc}$ & $88 \mathrm{a}$ \\
\hline 12 - Paraquat $48 \mathrm{~g} \mathrm{ha}^{-1}$ & $2.411 \mathrm{a}$ & $50,66 \mathrm{ab}$ & $86 \mathrm{a}$ \\
\hline CV \% & 13,77 & 34,12 & 3,39 \\
\hline Valor de F & 0,68 & 26,08 & 0,98 \\
\hline DMS & 889 & 18,92 & 13 \\
\hline
\end{tabular}

I/ Médias seguidas pela mesma letra não diferem entre si, a 5\% de probabilidade, pelo teste de Tukey.

Tabela 6 - Peso de espigas, peso de grãos e peso de 1.000 grãos para plantas de milho submetidas aos diferentes tratamentos estudados, no ensaio 1997/98. Embrapa Milho e Sorgo. Sete Lagoas-MG

\begin{tabular}{|l|c|c|c|}
\hline \multicolumn{1}{|c|}{ Tratamentos } & $\begin{array}{c}\text { Peso de } \\
\text { espigas } \\
\left(\mathrm{kg} \mathrm{ha}^{-1}\right)\end{array}$ & $\begin{array}{c}\text { Peso de } \\
\text { grãos } \\
\left(\mathrm{kg} \mathrm{ha}^{-1}\right)\end{array}$ & $\begin{array}{c}\text { Peso de } \\
1.000 \mathrm{grãos} \\
(\mathrm{g})\end{array}$ \\
\hline 1 - Testemunha & $7.986 \mathrm{ab}^{1 /}$ & $6.783 \mathrm{ab}$ & $229 \mathrm{a}$ \\
\hline 2 - Glyphosate $28,8 \mathrm{~g} \mathrm{ha}^{-1}$ & $8.038 \mathrm{a}$ & $6.830 \mathrm{ab}$ & $212 \mathrm{a}$ \\
\hline 3 - Glyphosate $57,6 \mathrm{~g} \mathrm{ha}^{-1}$ & $8.613 \mathrm{a}$ & $7.339 \mathrm{a}$ & $240 \mathrm{a}$ \\
\hline 4 - Glyphosate $86,4 \mathrm{~g} \mathrm{ha}^{-1}$ & $7.068 \mathrm{ab}$ & $6.008 \mathrm{bc}$ & $225 \mathrm{a}$ \\
\hline 5 - Glyphosate $115,2 \mathrm{~g} \mathrm{ha}^{-1}$ & $7.526 \mathrm{ab}$ & $6.404 \mathrm{abc}$ & $220 \mathrm{a}$ \\
\hline 6 - Glyphosate $172,8 \mathrm{~g} \mathrm{ha}^{-1}$ & $5.688 \mathrm{c}$ & $4.901 \mathrm{~d}$ & $214 \mathrm{a}$ \\
\hline 7 - Testemunhana com adjuvante & $7.781 \mathrm{ab}$ & $6.490 \mathrm{abc}$ & $221 \mathrm{a}$ \\
\hline 8 - Paraquat $8 \mathrm{~g} \mathrm{ha}^{-1}$ & $7.320 \mathrm{ab}$ & $6.626 \mathrm{abc}$ & $214 \mathrm{a}$ \\
\hline 9 - Paraquat $16 \mathrm{~g} \mathrm{ha}^{-1}$ & $7.953 \mathrm{ab}$ & $6.773 \mathrm{ab}$ & $235 \mathrm{a}$ \\
\hline 10 - Paraquat $24 \mathrm{~g} \mathrm{ha}^{-1}$ & $7.835 \mathrm{ab}$ & $6.650 \mathrm{abc}$ & $223 \mathrm{a}$ \\
\hline 11 - Paraquat $32 \mathrm{~g} \mathrm{ha}^{-1}$ & $7.527 \mathrm{ab}$ & $6.405 \mathrm{abc}$ & $226 \mathrm{a}$ \\
\hline 12 - Paraquat $48 \mathrm{~g} \mathrm{ha}^{-1}$ & $6.828 \mathrm{~b}$ & $5.775 \mathrm{c}$ & $217 \mathrm{a}$ \\
\hline CV \% & 9,41 & 9,13 & 8,46 \\
\hline Valor de F & 4,45 & 4,52 & 0,84 \\
\hline DMS & 1.767 & 1.464 & 47 \\
\hline
\end{tabular}

I/ Médias seguidas pela mesma letra não diferem entre si, a 5\% de probabilidade, pelo teste de Tukey.

A deriva do glyphosate em baixas concentrações (doses reduzidas de 2 a 4\%) não afetou o rendimento final de grãos. No entanto, derivas de glyphosate e paraquat a partir de $8 \%$ da dose recomendada podem influir no desenvolvimento das plantas, causando necroses na parte aérea e comprometendo a produtividade de grãos. O prejuízo da deriva simulada foi diretamente proporcional ao aumento da subdose dos produtos.

\section{AGRADECIMENTOS}

A José Eduardo Filho, Assistente de Pesquisa, e a José Rubens de Oliveira, Técnico Agrícola da Embrapa Milho e Sorgo, pela colaboração na coleta de dados.

\section{LITERATURA CITADA}

ALLISON, J.C.S.; WATSON, D.J. The production and distribution of dry matter maize after flowering. Ann. Bot., v.30, p.365-381, 1966.

ALI, M.; FLETCHER, R.A. Phytotoxic action of glyphosate and amitrole on corn seedlings. Can. J. Bot., v.56, p.2196-2202,1978.

BODE, L. E. Downwind drift deposits by ground applications. In: PROCEEDINGS OF PESTICIDE DRIFT MANAGEMENT SYMPOSIUM, 1984 , Brookings. Proceedings... S.1.: 1984. p.49-52.

HOAGLAND, R.E.; DUKE, S.O.; ELMORE, C.D. Effects of glyphosate on metabolism of phenolic compounds. III. Phenylalanine ammonium-lyase activity, free amino acids, soluble protein, and hydroxyphenolic componds in axes of dark-grown soybeans. Physiol. Plant., v.46, p.357-366, 1979.

MacKINNON, J.C. Energy allocation during growth of six maize hybrids in Nova Scotia. Can. J. Plant Sci., v.59, p.667-77, 1979.

MAGAlHÃES, P.C.; JONES, R. Aumento de fotoassimilados sobre os teores de carboidratos e nitrogênio em milho. Pesq. Agropec. Bras., v.25, n.12, p.1755-1761, 1990a.

MAGAlhães, P.C.; JONES, R. Aumento de fotoassimilados na taxa de crescimento e peso final dos grãos de milho. Pesq. Agropec. Bras., v.25, n.12, p.1747-1754, 1990b.

MAGAlHÃES, P.C.; DURÃES, F.O.M.; PAIVA, E. Fisiologia da planta de milho. Sete Lagoas: EMBRAPA-CNPMS, 1995. 27p. (EMBRAPACNPMS. Circular Técnica, 20). 
MAGALHÃES, P.C.; DURÃES, F.O.M.; OLIVEIRA, A.C. Efeitos do quebramento do colmo no rendimento de grãos de milho. Ci. Agrotecnol., v.22, n.3, p.279-289, 1998.

MAGALHÃES, P.C.; DURÃES, F.O.M.; OLIVEIRA, A.C.; GAMA, E.E.G. Efeitos de diferentes técnicas de despendoamento na produção de milho. Sci. Agríc., v.56, n.1, p.77-82, 1999.

MILHO: em grão - 1a safra. Levantamento Sistemático da Produção Agrícola, v.6, n.4, p.38-40, 1998.

PURÍSSIMO, C. Classificação de herbicidas quanto ao mecanismo de ação. In: SEMINÁRIO SOBRE SISTEMA PLANTIO DIRETO NA UFV, 2., 1999, Viçosa. Anais... Viçosa: UFV, 1999. p.73-110.
RESENDE, M.; FRANÇA, G.E.; ALBUQUERQUE, P.E.P.; ALVES, V.M.C. O momento de irrigar a cultura do milho de inverno na Região Sudeste. In: CONGRESSO NACIONAL DE MILHO E SORGO, 19; REUNIÃO TÉCNICA ANUAL DO MILHO, 37, REUNIÃO TÉCNICA ANUAL DO SORGO, 21, Porto Alegre, RS. Anais... Porto Alegre: SAA/SCT/ABMS/EMATER - RS/ CNPMS-EMBRAPA/CIENTEC, 1992. p.144.

SILVA, J.B.; RODRIGUES, M.A.T.; BEGLIOMINI, E. Indicações para o controle de plantas daninhas na cultura do milho em pós-emergência com o herbicida Sanson 40 SC e sua mistura 1+2 com atrazine. O Ruralista, v.35, n.440, p.9-11, 1998.

WALL, D. A. Potato (Solanum tuberosum) response to simulated drift of dicamba, clopyralid, and tribenuron. Weed Sci., v.42, p.110-114, 1994. 